\title{
3 Chronische Schmerztherapie bei Adipositas
}

\author{
Christoph H.R. Wiese und Christoph L. Lassen
}

In der klinischen Schmerzmedizin werden in aller Regel drei unterschiedliche Arten (akuter Schmerz, chronischer Schmerz, Tumorschmerz) von Schmerzen beschrieben, diagnostiziert und therapiert. Diese teilen sich zusätzlich in verschiedene Untergruppen auf, von denen jede für sich eine eigene individuelle, patientenadaptierte (Alter, Sozialstatus, Schmerzanamnese, Zielsetzung und Co-Morbiditäten) Therapieform beinhalten sollte. Neben der Therapie akuter Schmerzen spielt in der Schmerztherapie somit noch die Therapie von Tumorschmerzen und die Therapie von chronischen nicht malignen Schmerzen eine wichtige Rolle.

In der medikamentösen Tumorschmerztherapie gibt es kaum spezifische Unterschiede bezüglich der Therapie von adipösen und normalgewichtigen Patienten. Anders ist dies sowohl in der medikamentösen als auch nicht medikamentösen Therapie chronischer nicht maligner Schmerzen. Hier muss ein besonderes Augenmerk auf die Situation adipöser Patienten, unter Berücksichtigung sowohl der körperlichen als auch der seelischen Verfassung, geworfen werden.

Daher erfolgt in diesem Kapitel insbesondere die Darstellung der Therapie nicht maligner chronischer Schmerzen.

Im Gegensatz zum akuten Schmerz hat der chronische Schmerz seine biologische Warnfunktion verloren. Neuere Definitionen chronischer Schmerzgeschehen beziehen sich nicht mehr ausschließlich auf eine zeitliche Dauer, sondern beinhalten auch die psychosozialen Begleitumstände der Erkrankung. Chronische Schmerzerkrankungen zeigen sich dementsprechend in mehreren Lebenssituationen der Patienten. Sie beeinträchtigen die körperliche Integrität, das seelische Befinden und die sozialen Beziehungen (beruflich und privat). Man spricht auch vom zugrundeliegenden biopsychosozialen Krankheitsmodell. Eine rein somatisch orientierte Therapie kann somit nur in den seltensten Fällen zu einem dauerhaften und zufriedenstellenden therapeutischen Erfolg führen. In der Regel ist eine interdisziplinäre Behandlung unter Einbeziehung verschiedener Therapierichtungen (medikamentöse Therapie, interventionelle Therapie, Physiotherapie, Schmerzpsychotherapie) notwendig. Dieses therapeutische Prinzip ist bei adipösen Patienten wie bei Normalgewichtigen anzuwenden. Somit führt beim Problem Schmerz bzw. der Diagnose "chronischer Schmerz" eine reine Gewichtsreduktion, z.B. durch eine bariatrische Operation, in den meisten Fällen nicht zu einer klinisch relevanten Schmerzreduktion und somit auch nicht zum gewünschten Therapieerfolg (Cremieux et al. 2010).

Auch bei adipösen Patienten ist ein biopsychosozia-
ler Ansatz in der Therapie chronischer Schmerzen
von hoher Bedeutung. Eine Reduktion der komple-
xen Gesamterkrankung sollte nicht auf eine reine Ge-
wichtsverminderung reduziert werden.

\subsection{Grundsätze}

Die Chronifizierung von Schmerzen geht oftmals mit einer Passivität der Patienten einher. Diese ist häufig mit einer erhöhten Anspruchshaltung an den Therapeuten verbunden, der „etwas“ machen soll, z.B. 
Medikamente verschreiben, Injektionen verabreichen oder operieren. Die Ursache der Beschwerden wird meist in körperlichem/somatischem Ursprung gesehen. Die daraus resultierenden Schmerzen werden insgesamt als unbeeinflussbar empfunden, und somit werden therapeutische/aktivierende Maßnahmen als uneffektiv und nicht heilungsfördernd klassifiziert. Hierdurch erhöht der Patient sein passives Verhalten zunehmend.

Diese Grundhaltung gilt auch für viele Patienten mit Adipositas (,Sport ist Mord“). Der körperliche Zustand wird oft als unbeeinflussbar gesehen bzw. vorherige Abnehmversuche haben zu keiner Besserung geführt („Ich esse doch fast nichts“). Daher gilt gerade für die Behandlung von adipösen chronischen Schmerzpatienten, dass zu Behandlungsbeginn dezidiert geklärt werden muss, dass der Patient eine aktive Rolle in der Therapie übernimmt und die Therapeuten insgesamt nur unterstützend wirken. Weiterhin muss mit dem Patienten besprochen werden, dass eine Cewichtsreduktion der Erzielung eines dauerhaften Therapieerfolgs förderlich ist. Ob diese Gewichtsreduktion parallel zur Schmerztherapie erfolgen soll oder eine Vorbedingung zur Aufnahme einer Therapie ist bleibt zu diskutieren (Aronoff 2009). Entscheidend ist es, dem Patienten zu vermitteln, dass seine Beschwerden sehr wohl beeinflussbar sind. Das Essverhalten eines Patienten, die ebenso vorhandene Körperwahrnehmung und die Bereitschaft in diesen Bereichen etwas verändern zu wollen, hat einen sehr hohen Einfluss auf den schmerztherapeutischen Erfolg. Hierbei ist es primär nicht von Bedeutung, ob beispielsweise Wirbelsäulenbeschwerden durch das Gewicht beeinflusst werden oder nicht. Die Crundeinstellung des Patienten muss sich im Rahmen der Therapie entscheidend ändern, will er langfristig auch schmerztherapeutisch einen Erfolg erzielen. Es ist also in der Therapie von besonderer Bedeutung, dass der Patient die Probleme, die mit seiner Adipositas einhergehen, erkennt und auch bereit ist, diese zu behandeln bzw. zu lösen.

Das primäre Assessment des Patienten (durch Ärzte, Schmerzpsychologen, Physiotherapeuten) entscheidet im Verlauf über die weitere Therapieausrichtung und deren Form. Je nach Schwere des Beschwerdebildes kann eine Therapie ambulant (Schmerzambulanz, niedergelassene Schmerztherapeuten), tagesstationär (Schmerztagesklinik) oder vollstationär (Schmerzzentrum) erfolgen.

\subsection{Schmerzerkrankungen bei Adipösen}

Im Vordergrund chronischer Schmerzerkrankungen stehen, wie auch bei Normalgewichtigen, muskuloskelettale Erkrankungen. Insbesondere betroffen sind die Gelenke der unteren Extremität, z.B. Knie oder Hüfte. Häufig finden sich auch unspezifische Rückenbeschwerden, hauptsächlich der unteren Lendenwirbelsäule (LWS) (Urquhart et al. 2011). Es soll auch eine Assoziation zwischen dem Krankheitsbild der Fibromyalgie und dem Grad der Adipositas der Patienten bestehen (Okifuji et al. 2010). Gegebenenfalls kommt es bei adipösen Patienten im Verlauf der Zeit auch zu somatisch begründbaren Schmerzen aufgrund von Folgeerkrankungen der Adipositas wie z.B. schmerzhafter diabetischer Polyneuropathie, neuropathischer Schmerzen nach Wundheilungsstörungen und/oder Ulkusschmerzen am Unterschenkel.

\subsection{Therapiemöglichkeiten}

Die Therapie von chronischen Schmerzpatienten mit Adipositas sollte multimodal erfolgen. Dabei ist die medikamentöse Therapie (wie auch bei Normalgewichtigen) nur einer von vielen Bausteinen. Mindestens gleichbedeutend sind aktivierende Maßnahmen wie Ausdauersport und Physiotherapie sowie eine schmerzpsychologische Therapie, kombiniert mit Maßnahmen zur Gewichtsreduktion. Die einzelnen Therapieformen werden im Folgenden kurz dargestellt.

\subsubsection{Medikamentöse Therapiekomponente}

\section{Nicht-Opioid-Analgetika}

Unter diese Gruppe fallen die gängigen nicht-steroidalen Antirheumatika (NSAR), Paracetamol, Metamizol und die Coxibe. Eine Dauereinnahme von Nicht-Opioid-Analgetika wird in der Regel aufgrund der Nebenwirkungen weder bei adipösen noch bei normalgewichtigen Schmerzpatienten empfohlen (s. Tab. 3).

\section{Opioid-Analgetika}

Der Einsatz von Opioiden in der Langzeittherapie von Schmerzen, die keine maligne Ursache haben, wird in den letzten Jahren kritisch diskutiert. In der ak- 
Tab. 3 Beispiele für Nicht-Opioid-Analgetika

\begin{tabular}{|lll}
\hline Medikament & Nebenwirkung bei Dauereinnahme & Kommentar \\
\hline NSAR & $\begin{array}{l}\text { Magenulcus, Niereninsuffizienz, } \\
\text { Herz-Kreislauferkrankungen }\end{array}$ & hohes Nebenwirkungsrisiko \\
\cline { 2 - 3 } Paracetamol & Leberinsuffizienz & ungeeignet bei Leberschädigung (z.B. Fettleber) \\
\cline { 2 - 3 } Metamizol & Agranulozytose, Leber- und Niereninsuffizienz & $\begin{array}{l}\text { Agranulozytoserisiko wohl überschätzt (1:1.000.000), } \\
\text { Organtoxizität erst bei ausgeprägter Vorschädigung }\end{array}$ \\
\hline Coxibe & Herz-Kreislauferkrankungen & $\begin{array}{l}\text { höhere Herz-Kreislaufrisiken als NSAR, geringere } \\
\text { Magenschleimschädigung }\end{array}$ \\
\hline
\end{tabular}

tuellen Diskussion wird eher zum zurückhaltenden Einsatz tendiert (Reinecke u. Sorgatz 2009). Grund hierfür ist das nicht unerhebliche Missbrauchsrisiko dieser Substanzen, die Gefahr einer Überdosierung (Bohnert et al. 2011) verbunden mit der Annahme, dass psychische Störungen (Depression, Angststörungen) durch Opioide verstärkt werden. Dies gilt sowohl bei adipösen als auch bei normalgewichtigen Schmerzpatienten.

Kann bei adipösen Patienten auf Opioid-Analgetika nicht verzichtet werden, sollte nach pharmakokinetischen und-dynamischen Gesichtspunkten ausgewählt werden. Transdermale Applikationssysteme sind hierbei eher ungeeignet, da sich das Opioid im subkutanen Fettgewebe anreichern und die Aufnahme in das Blut bei großen subkutanen Fettmengen unkalkulierbar sein kann. Unter den oralen Opioiden erscheint Hydromorphon wegen der Unabhängigkeit des Stoffwechselweges von der Leberfunktion als relativ gut geeignet bei adipösen Patienten. Insgesamt wird die orale retardierte Medikation bevorzugt eingesetzt. Es erfolgt auch bei adipösen Patienten eine Dosierung nach der Wirkung und nicht nach dem absoluten Gewicht. Auch werden eventuelle Höchstdosierungen nicht überschritten.

\section{Antidepressiva}

Bei chronischen Schmerzerkrankungen gehören Antidepressiva und hierbei insbesondere die Gruppe der trizyklischen Antidepressiva zu den am häufigsten verschriebenen Medikamenten. Die größte Erfahrung und die meisten Wirksamkeitsnachweise gibt es für Amitriptylin, wobei auch andere trizyklische Antidepressiva bei chronischen Schmerzen wirksam sind. Eine genaue Patienteninformation zur Medikamentencompliance ist sowohl bei adipösen als auch bei normalgewichtigen Patienten notwendig, da gerade Antidepressiva häufig von Patienten abgelehnt werden, da „ihr persönliches Krankheitsbild“ keine Depression beinhaltet. Die Klärung der Ursache der Verschreibung ist sicher hilfreich für die weitere Therapie. Antidepressiva werden in der Schmerztherapie in der Regel nicht zur Therapie psychischer Erkrankungen eingesetzt, sondern zur Schmerzdistanzierung und zur Veränderung der Schmerzwahrnehmung. Dies muss dem Patienten vermittelt werden. Selten werden Dosierungen wie in der Depressionsbehandlung erreicht. Die Eindosierung von z.B. Amitriptylin sollte langsam erfolgen, beginnend mit 10-25 mg zur Nacht. Der Patient muss wissen, dass eine Wirkung frühestens nach 2-4 Wochen zu erwarten ist; unerwünschte Arzneimittelwirkungen (UAW) treten jedoch schon früher auf. Hierzu gehören neben ZNS-Störungen (Müdigkeit, Schwindel, Konzentrationsstörungen), Mundtrockenheit und nicht zuletzt eine manchmal deutlich ausgeprägte Gewichtszunahme. Dies schränkt den Einsatz dieser sonst gut wirksamen Medikamentengruppe bei adipösen Patienten leider häufig ein, wenn diese nicht zu aktivierenden Maßnahmen bereit sind. In diesem Zusammenhang empfiehlt sich auch vor Beginn der medikamentösen Therapie eine deutliche Gewichtsabnahme als Folge einer Änderung des Ess- und Wahrnehmungsverhaltens.

Außer den Trizyklika haben weitere Antidepressiva keinen so hohen Stellenwert in der Therapie chronischer Schmerzen. Als Ausnahmen sind jedoch noch Duloxetin für die Therapie der schmerzhaften diabetischen Polyneuropathie und einzelne SSRI (selektive Serotonin Wiederaufnahmehemmer) in der Therapie des Fibromyalgiesyndroms, wobei Duloxetin auch bei anderen chronischen Schmerzerkrankungen Anwendung findet. Duloxetin kann bei adipösen Patienten eine Alternative zu der Gruppe der Trizyklika darstellen, da die Gewichtszunahme nicht 
so ausgeprägt ist. Es ist allerdings auch hier auf einen erhöhten Aktivitätsgrad der Patienten zu achten. Eine reine passive Medikamentenkonsumierung ist sicher das falsche Signal.

\section{Antikonvulsiva}

Antikonvulsiva werden zunehmend häufig in der Therapie von chronischen Schmerzen eingesetzt, speziell bei Schmerzen mit neuropathischer Komponente. Neben den klassischen neuropathischen Schmerzerkrankungen, wie z.B. postherpetische Neuralgie oder die schmerzhafte diabetische Polyneuropathie, gehören hierzu auch unspezifische Rückenschmerzen, für die eine neuropathische Komponente in immerhin 33\% der Patienten angenommen wird (Freynhagen et al. 2006).

Problematisch an den meisten Antikonvulsiva sind die UAW, die neben ZNS-Problemen, Leberfunktionseinschränkungen nicht zuletzt ebenfalls wie bei den trizyklischen Antidepressiva eine Gewichtszunahme miteinschließen. Die Konsequenzen sind oben bereits beschrieben und gelten für die Antikonvulsiva ebenso. Einen Überblick über schmerztherapeutisch häufig eingesetzte Antikonvulsiva bietet Tabelle 4.

Auch die Antikonvulsiva werden wirkungsadaptiert sowohl bei adipösen als auch bei normalgewichtigen Patienten eingesetzt. Eine Orientierung am absoluten Körpergewicht findet bei beiden Patientengruppen nicht statt.

\subsubsection{Psychotherapeutische Komponente}

Die schmerzpsychologische Therapie bei chronischen Schmerzpatienten umfasst einerseits eine spezifisch auf die Schmerzen zielende Therapie, welche sich von der normalgewichtiger Patienten in der Regel nicht unterscheidet (Schmerzedukation, Achtsamkeitsübungen, Biofeedback etc.). Andererseits umfasst sie aber auch eine allgemeine psychologische Therapie bestehender psychischer Komorbiditäten im Rahmen der Adipositas als Krankheitsbetrachtung, da sich bei adipösen Patienten gehäuft psychische Komorbiditäten erkennen lassen, die in dieser Spezifität bei normalgewichtigen Patienten nicht bestehen. Die Prävalenz psychischer Komorbiditäten, die auch einen Einfluss auf die Schmerzerkrankung aufweisen, nimmt mit steigendem BMI zu (Heo et al. 2006). Im Vordergrund stehen hierbei Depression, Angststörungen und Essstörungen .

Weiterhin gehört es zu der schmerzpsychologischen Therapie, die Patienten immer wieder zu motivieren sowie Gewichtsreduktion und aktivierende Therapie fortzuführen. Diese Motivation muss natürlich durch alle beteiligten Behandler erfolgen und ist nicht nur auf die Schmerzpsychologen beschränkt. Besondere Rücksicht gilt es auch auf die entsprechenden Co-Morbiditäten adipöser Patienten zu nehmen. Hier sind insbesondere psychische Begleiterkrankungen zu nennen, die es im Rahmen der Schmerztherapie mitzubeachten und ggf. zu behandeln gilt. Auf mögliche Zielkonflikte sollte der Therapeut gerade bei adipösen Patienten ebenfalls achten (beispielsweise Schmerz als Rechtfertigung für

Tab. 4 Überblick über Antikonvulsiva zur Schmerztherapie

\begin{tabular}{|c|c|c|}
\hline Medikament & Dosierung & Bemerkungen \\
\hline Pregabalin & $\begin{array}{l}25 \mathrm{mg}-0 \mathrm{mg}-25 \mathrm{mg} \text {, } \\
\text { Steigerung alle } 3-5 \text { Tage um } 25 \mathrm{mg} \text { Einzeldosis, } \\
\text { max. } 300 \mathrm{mg}-0 \mathrm{mg}-300 \mathrm{mg}\end{array}$ & $\begin{array}{l}\text { breiter Einsatz bei verschiedensten neuro- } \\
\text { pathischen Schmerzerkrankungen, vergleichsweise } \\
\text { gute Verträglichkeit bei langsamer Aufdosierung }\end{array}$ \\
\hline Gabapentin & $\begin{array}{l}100 \mathrm{mg}-100 \mathrm{mg}-100 \mathrm{mg} \text {, } \\
\text { Steigerung alle } 3-5 \text { Tage um } 100 \mathrm{mg} \text { Einzeldosis, } \\
\text { max. } 1.200 \mathrm{mg}-1.200 \mathrm{mg}-1.200 \mathrm{mg}\end{array}$ & $\begin{array}{l}\text { breiter Einsatz bei verschiedensten neuro- } \\
\text { pathischen Schmerzerkrankungen, vergleichsweise } \\
\text { gute Verträglichkeit bei langsamer Aufdosierung }\end{array}$ \\
\hline Carbamazepin & $\begin{array}{l}200 \mathrm{mg} \text { retard abends, } \\
\text { bis max. } 400 \mathrm{mg}-0 \mathrm{mg}-400 \mathrm{mg}\end{array}$ & $\begin{array}{l}\text { Mittel der } 1 \text {. Wahl bei Gesichtsneuralgien, lange } \\
\text { erprobtes Medikament, viele UAW (ZNS, Leber) }\end{array}$ \\
\hline Topiramat & $\begin{array}{l}25 \mathrm{mg} \text { abends, } \\
\text { Aufdosierung in } 25 \mathrm{mg} \text {-Schritten jeweils über eine } \\
\text { Woche, } \\
\text { Zieldosis } 100-200 \mathrm{mg} \text {, max. } 500 \mathrm{mg} \text { auf zwei Einzeldosen }\end{array}$ & $\begin{array}{l}\text { geringere Wirksamkeit als die anderen } \\
\text { Antiepileptika bei neuropathischen Schmerzen, } \\
\text { Gewichtsabnahme als weitere Wirkung }\end{array}$ \\
\hline
\end{tabular}


Essen und somit auch für Inaktivität und Gewichtszunahme).

\subsubsection{Ernährungstherapeutische Komponente}

Die wenigsten adipösen Patienten schaffen eine dauerhafte Gewichtsreduktion ohne weitere professionelle Hilfen. Für die gesamte Therapie von adipösen Schmerzkranken gilt, dass ein langfristiger Therapieerfolg nur bei ausreichender Eigenmotivation der Patienten möglich ist. Dies gilt insbesondere für den Bereich der Ernährungstherapie/Gewichtsabnahme. Diese zielt nicht nur auf eine reine Gewichtsabnahme hin, sondern insbesondere auch auf eine generelle Änderung der Lebensweise der Patienten. Es sollten mehrere Ansätze kombiniert und unter Berücksichtigung der einzelnen Lebensumstände individuell auf den Patienten zugeschnitten werden. In diesem Zusammenhang sind im Vergleich zu normalgewichtigen Patienten Ernährungsberater im multimodalen schmerztherapeutischen Team unabdingbar und sollten als einheitlicher Therapiestandard gefordert werden. Diese können natürlich wie alle schmerztherapeutischen Programme nur Anstöße für eine gesamte Änderung der Lebenssituation sein. Der Patient muss sich darauf einlassen und sollte, sofern eine eindeutige Compliance nicht erkennbar oder nicht gewollt ist, auch nicht in derartige Schmerzprogramme integriert werden, da hier die Zielsetzungen zwischen Therapeuten und Patienten deutlich divergieren und somit die therapeutischen Versuche in aller Regel durch gegenseitige Frustration geprägt sind. Von den folgenden ernährungstherapeutischen Maßnahmen sollten mit dem Patienten zusammen die für ihn passenden gewählt werden. Idealerweise wird mit der Ernährungstherapie schon vor dem Beginn der Schmerztherapie begonnen.

- eigenständige Diäten (z.B. nach den Leitlinien der deutschen Adipositasgesellschaft),

- psychologisch begleitete Esstherapie,

- medikamentöse Maßnahmen (z.B. Sibutramin, Orlistat),

- Gewichtsreduktionsprogramme: ärztlich geführt (Docweight, Optifast) oder Selbsthilfe-Programme (z.B. WeightWatchers),

- operative Maßnahmen.

\subsubsection{Physiotherapeutische Komponente}

Die Physiotherapie stellt einen zentralen Pfeiler bei der Behandlung von adipösen Schmerzpatienten dar. Dabei verfolgt die Physiotherapie mehrere Ziele. Zum einen soll über eine Aktivitätserhöhung ein erhöhter Kalorienverbrauch und damit eine negative Kalorienbilanz erzielt werden. Andererseits kommen physiotherapeutische Maßnahmen zur direkten Schmerzreduktion zum Einsatz. Aktivierenden Maßnahmen (Krankengymnastik, Übungen am Gerät) ist insgesamt bei allen Patienten, aber insbesondere bei adipösen Patienten, Vorzug vor passiven Maßnahmen (Massagen, Fango) zu geben.

Die physiotherapeutischen Übungen sollten individuell nach den Möglichkeiten des Patienten gewählt werden. Können z.B. Übungen im Stehen wegen Knieschmerzen nicht durchgeführt werden, sind die Übungen vielleicht im Liegen oder Sitzen möglich. Als besonders hilfreich empfinden viele Patienten Gymnastik im Wasser, da hier die Gelenke entlastet werden.

Neben krankengymnastischen Übungen sollten die Patienten auch moderaten aeroben Ausdauersport betreiben. Empfohlen werden hier z.B. Schwimmen oder Nordic walking auf weichem Untergrund (2-3 $\mathrm{x}$ pro Woche, 30-6o min).

\subsubsection{Therapiebeispiel für ein multimodales Konzept}

In Tabelle 5 ist ein 4-wöchiges tagesstationäres Konzept dargestellt, das bei adipösen Schmerzpatienten Anwendung finden kann. Die medikamentöse Komponente wurde aufgrund ihrer Individualität in der Erscheinungsform und somit in der Therapie des einzelnen Patienten hierbei nicht berücksichtigt. Vor der Aufnahme einer tagesstationären Behandlung muss der Patient von allen beteiligten Fachdisziplinen evaluiert werden. Im Vordergrund steht der Wille, an den Therapiemaßnahmen aktiv teilzunehmen und tatsächlich zu einer langfristigen Änderung der Lebensweise bereit zu sein. Dementsprechend schließt sich an eine tagesstationäre Therapie eine intensive ambulante Weiterbetreuung von mindestens einem Jahr an.

\section{Fazit für die Praxis}

Die Schmerztherapie bei adipösen Patienten mit chronischen Schmerzen muss multimodal unter Einschluss von Ernährungstherapeuten erfolgen. Sie umfasst neben der rei- 
Tab. 5 Beispiel für einen Therapieplan „Chronische Schmerztherapie“ bei adipösen Patienten

\begin{tabular}{|c|c|c|c|c|c|}
\hline Uhrzeit & Montag & Dienstag & Mittwoch & Donnerstag & Freitag \\
\hline $8-9$ & $\begin{array}{l}\text { gemeinsames } \\
\text { Frühstück }\end{array}$ & $\begin{array}{l}\text { gemeinsames } \\
\text { Frühstück }\end{array}$ & $\begin{array}{l}\text { gemeinsames } \\
\text { Frühstück }\end{array}$ & $\begin{array}{l}\text { gemeinsames } \\
\text { Frühstück }\end{array}$ & $\begin{array}{l}\text { gemeinsames } \\
\text { Frühstück }\end{array}$ \\
\hline $9-10$ & $\begin{array}{l}\text { Gruppensport } \\
\text { (Bewegungsbad) }\end{array}$ & $\begin{array}{l}\text { Gruppensport } \\
\text { (Gymnastik) }\end{array}$ & $\begin{array}{l}\text { Gruppensport } \\
\text { (Bewegungsbad) }\end{array}$ & $\begin{array}{l}\text { Gruppensport } \\
\text { (Gymnastik) }\end{array}$ & $\begin{array}{l}\text { Gruppensport } \\
\text { (Bewegungsbad) }\end{array}$ \\
\hline $10-11$ & $\begin{array}{l}\text { Gruppentherapie } \\
\text { Schmerzedukation }\end{array}$ & $\begin{array}{l}\text { Gruppentherapie } \\
\text { (Entspannung) }\end{array}$ & $\begin{array}{l}\text { Gruppentherapie } \\
\text { (Entspannung) }\end{array}$ & $\begin{array}{l}\text { Gruppentherapie } \\
\text { Schmerzedukation }\end{array}$ & $\begin{array}{l}\text { Gruppentherapie } \\
\text { (Entspannung) }\end{array}$ \\
\hline $11-13$ & $\begin{array}{l}\text { Mittagessen mit } \\
\text { Ernährungsberatung }\end{array}$ & $\begin{array}{l}\text { Mittagessen mit } \\
\text { Ernährungsberatung }\end{array}$ & $\begin{array}{l}\text { Mittagessen mit } \\
\text { Ernährungsberatung }\end{array}$ & $\begin{array}{l}\text { Mittagessen mit } \\
\text { Ernährungsberatung }\end{array}$ & $\begin{array}{l}\text { Mittagessen mit } \\
\text { Ernährungsberatung }\end{array}$ \\
\hline $13-14$ & $\begin{array}{l}\text { Einzeltherapie } \\
\text { (ärztlich) }\end{array}$ & $\begin{array}{l}\text { Einzeltherapie } \\
\text { (psychologisch) }\end{array}$ & $\begin{array}{l}\text { Einzeltherapie } \\
\text { (ärztlich) }\end{array}$ & $\begin{array}{l}\text { Einzeltherapie } \\
\text { (psychologisch) }\end{array}$ & $\begin{array}{l}\text { Einzeltherapie } \\
\text { (psychologisch) }\end{array}$ \\
\hline $14-15$ & $\begin{array}{l}\text { Sporttherapie } \\
\text { (Gerät) }\end{array}$ & $\begin{array}{l}\text { Sporttherapie } \\
\text { (Schwimmen) }\end{array}$ & $\begin{array}{l}\text { Sporttherapie } \\
\text { (Nordic walking) }\end{array}$ & $\begin{array}{l}\text { Sporttherapie } \\
\text { (Schwimmen) }\end{array}$ & $\begin{array}{l}\text { Sporttherapie } \\
\text { (Gerät) }\end{array}$ \\
\hline $15-16$ & $\begin{array}{l}\text { Ergotherapie/ } \\
\text { work hardening }\end{array}$ & $\begin{array}{l}\text { Gruppentherapie } \\
\text { Schmerzedukation }\end{array}$ & Ergotherapie & $\begin{array}{l}\text { Ergotherapie/ } \\
\text { work hardening }\end{array}$ & Ergotherapie \\
\hline
\end{tabular}

nen Schmerztherapie eine parallele, bzw. schon im Vorfeld stattgehabte Gewichtsreduktion und ernährungstherapeutische Komponente. In dieser Hinsicht unterscheidet sich die Therapie adipöser Patienten von derjenigen bei Normalgewichtigen. Die Therapie, soll sie denn erfolgreich sein, erfordert vom Patienten ein hohes Maß an Selbstdisziplin, Aktivität, Teilnahme und Eigenverantwortung. Die Therapie komplexer Erkrankungen (hohe Schmerzintensität, psychische Komorbidität, hoher BMI) sollte aufgrund der hohen Anforderung an die Behandler tages- oder vollstationär durchgeführt werden.

\section{Literatur}

Aronoff G (2009) Chronic pain, smoking, and obesity: a pain physician's perspective on patient selection. Pain Med 10, 962-965

Bohnert AS, Valenstein M, Bair M), Ganoczy D, McCarthy JF, Ilgen MA, Blow FC (2011) Association between opioid prescribing patterns and opioid overdose-related deaths. JAMA 305, 1315-1321

Cremieux PY, Ledoux S, Clerici C, Cremieux F, Buessing M (2010) The impact of bariatric surgery on comorbidities and medication use among obese patients. Obes Surg 20, 861-870
Freynhagen R, Baron R, Tolle T, Stemmler E, Gockel U, Stevens M, Maier C (2006) Screening of neuropathic pain components in patients with chronic back pain associated with nerve root compression: a prospective observational pilot study (MIPORT). Curr Med Res Opin 22, 529-537

Hauner H, Buchholz G, Hamann A, Husemann B, Koletzko B, Liebermeister H, Wabitsch M, Westenhöfer J, Wirth A (2007) Prävention und Therapie der Adipositas.

http://www.adipositas-gesellschaft.de/fileadmin/PDF/ Leitlinien/Adipositas-Leitlinie-2007.pdf, abgerufen am 07. Januar 2012

Heo M, Pietrobelli A, Fontaine KR, Sirey JA, Faith MS (2006) Depressive mood and obesity in US adults: comparison and moderation by sex, age, and race. Int I Obes 30, 513-519

Okifuji A, Donaldson GW, Barck L, Fine PG (2010) Relationship between fibromyalgia and obesity in pain, function, mood, and sleep. I Pain 11, 1329-1337

Reinecke H, Sorgatz H (2009) S3-Leitlinie LONTS Langzeitanwendung von Opioiden bei nicht tumorbedingten Schmerzen. Schmerz 23, 440-447

Urquhart DM, Berry P, Wluka AE, Strauss B|, Wang Y, Proietto I, Jones G, Dixon JB, Cicuttini FM (2011) Increased fat mass is associated with high levels of low back pain intensity and disability. Spine (Phila Pa 1976) 36, 1320-1325 


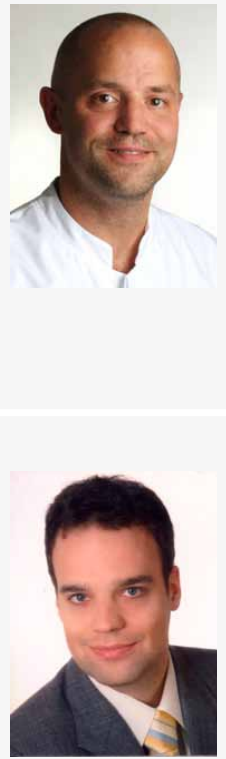

\section{PD Dr. med. Christoph H.R. Wiese}

Nach dem Medizinstudium an der Georg-Augustin-Universität Göttingen Beginn der klinischen Tätigkeit 1999 am Klinikum Braunschweig in der Klinik für Anästhesiologie. Von 20022008 Arzt in Weiterbildung am Uniklinikum Göttingen. 2002 Promotion. Ab 2004 Facharzt für Anästhesiologie und Erlangung der Zusatzbezeichnungen Notfallmedizin, Intensivmedizin, spezielle Schmerztherapie und Palliativmedizin. Seit 2009 Oberarzt der Klinik für Anästhesiologie am Uniklinikum Regensburg sowie Leiter der interdisziplinären Schmerzambulanz. 2010 Habilitation zum Thema „Palliative Notfälle - eine neue Herausforderung für die Notfallmedizin“.

\section{Dr. med. Christoph L. Lassen}

Nach dem Medizinstudium an der Christian-Albrechts-Universität Kiel mit Forschungsaufenthalt an der University of Utah in Salt Lake City, USA, arbeitete er seit 2005 als Arzt in Weiterbildung am Universitätsklinikum Regensburg in der Klinik für Anästhesiologie. 2009 Promotion. Besondere Interessensschwerpunkte sind Schmerztherapie und Palliativmedizin. 